\title{
Radiopharmaceutical Compound
}

National Cancer Institute

\section{Source}

National Cancer Institute. Radiopharmaceutical Compound. NCI Thesaurus. Code C1446.

An agent that contains a radioactive isotope and is used for diagnostic or therapeutic purposes. 